Numerical Electromagnetics Simulations of the Leakage Through the Pump-out Holes in the DISC Electromagnetic Interference Shield

C. G. Brown, A. Cooper, A. S. Moore

October 11, 2010 
This document was prepared as an account of work sponsored by an agency of the United States government. Neither the United States government nor Lawrence Livermore National Security, LLC, nor any of their employees makes any warranty, expressed or implied, or assumes any legal liability or responsibility for the accuracy, completeness, or usefulness of any information, apparatus, product, or process disclosed, or represents that its use would not infringe privately owned rights. Reference herein to any specific commercial product, process, or service by trade name, trademark, manufacturer, or otherwise does not necessarily constitute or imply its endorsement, recommendation, or favoring by the United States government or Lawrence Livermore National Security, LLC. The views and opinions of authors expressed herein do not necessarily state or reflect those of the United States government or Lawrence Livermore National Security, LLC, and shall not be used for advertising or product endorsement purposes.

This work performed under the auspices of the U.S. Department of Energy by Lawrence Livermore National Laboratory under Contract DE-AC52-07NA27344. 


\title{
Numerical Electromagnetics Simulations of the Leakage Through the Pump-out Holes in the DISC Electromagnetic Interference Shield
}

\author{
Charles G. Brown Jr., Amy Cooper, and Alastair S. Moore
}

October 8, 2010

\section{Background}

In order to prevent electromagnetic interference (EMI) from affecting the DISC diagnostic, an EMI shield was added. Figure 1 is a cross section from a CAD model of DISC and shows the EMI shield in situ. The shield is orange and at the top of the figure. Figure 2 is a drawing of just the EMI shield. The slit in the center of the EMI shield is covered by a metal mesh, which is not shown in this drawing. The small holes toward the base of the conical portion of the EMI shield are the pump-out holes, and the electromagnetic leakage through these holes is the subject of this report ${ }^{1}$. An alternate design for the EMI shield is considered in order to determine how to increase the EMI effectiveness of the pump-out holes in the shield without compromising the flow rate through the shield. Both the original and alternate designs are simulated and compared.

\section{Simulations}

Two types of electromagnetic wave incidence are considered in the simulations of the original and alternate designs in this report: longitudinal and transverse. In longitudinal incidence, the electromagnetic waves strike the EMI shield head on. In transverse incidence, the waves impinge on the EMI shield radially. The simulations of the original and alternate designs for both of these cases are described in the following subsections. All simulations are performed using $\mathrm{HFSS}^{2}$, version 12.1.1.

\footnotetext{
${ }^{1}$ In the models in this report, all of the other possible leakage paths are sealed so that the numerical electromagnetics simulations pertain only to the leakage through the pump-out holes.

${ }^{2}$ [http://www.ansoft.com/products/hf/hfss/] (Accessed August, 2010)
} 


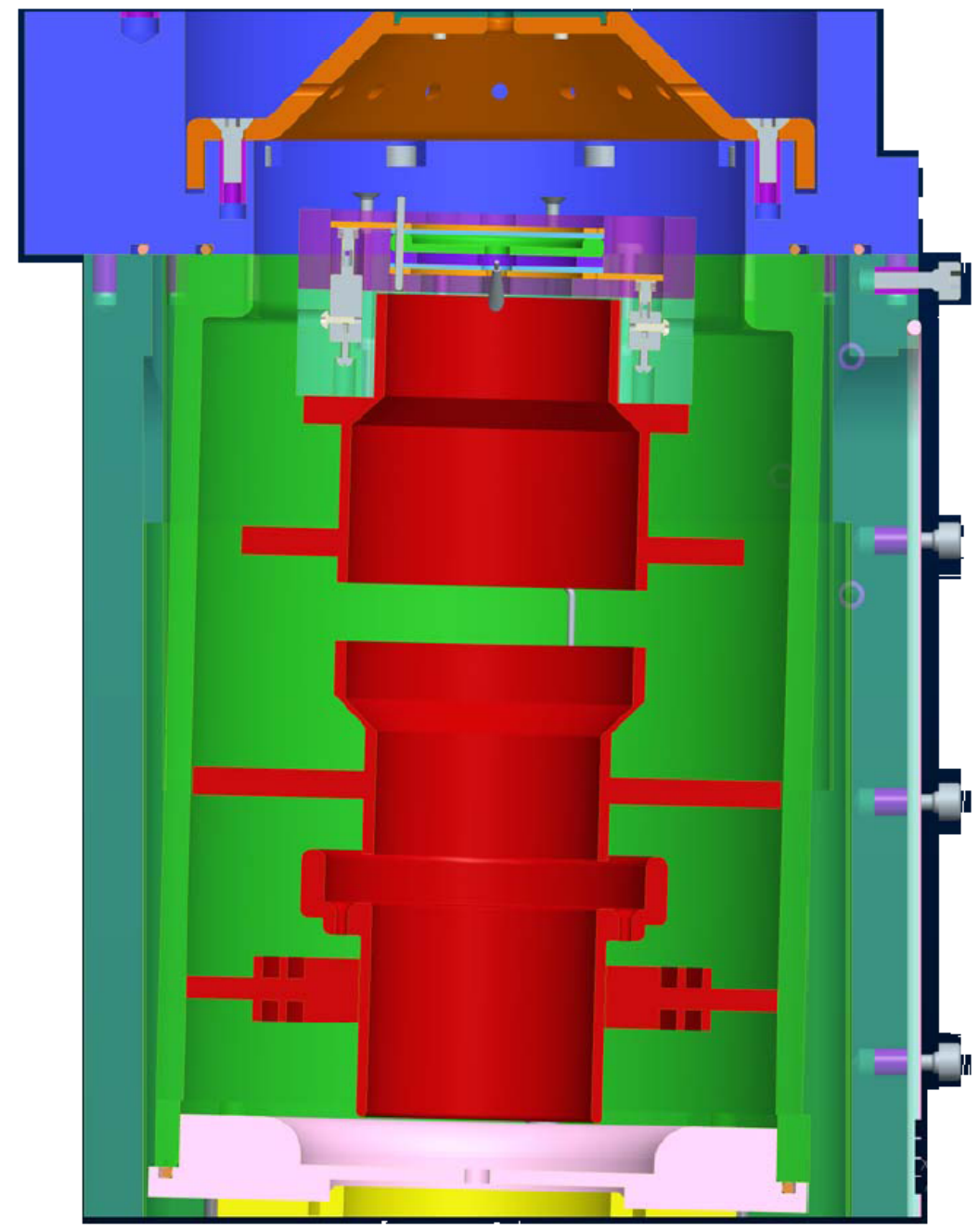

Figure 1: Cross section from a CAD model of DISC showing the EMI shield in situ. The shield is orange and at the top of the figure. 


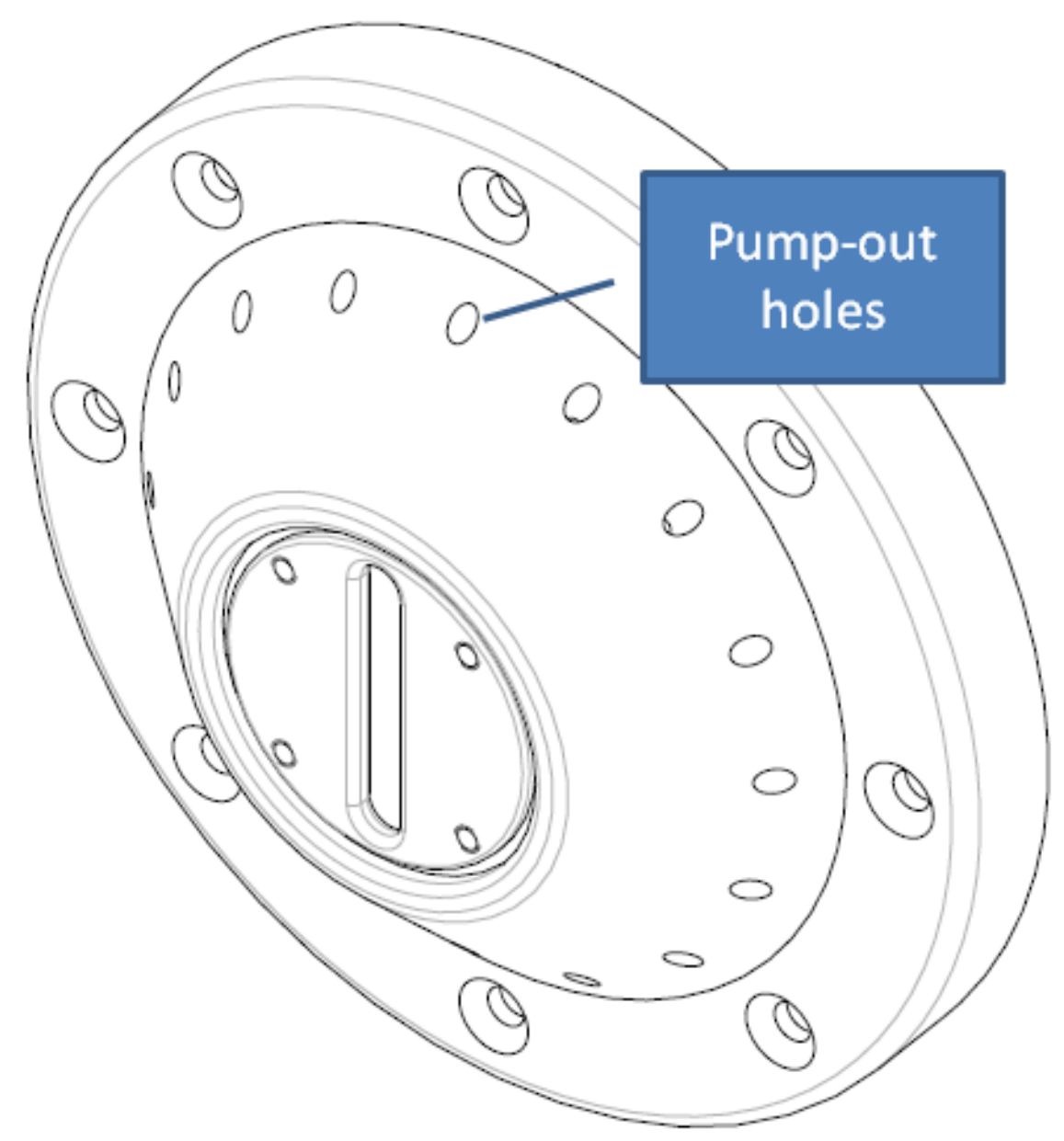

Figure 2: Drawing of the DISC EMI shield. 


\subsection{Longitudinal Incidence}

First, longitudinal incidence is examined for both the original and alternate designs (Figs. 3 and 4, respectively). The gray cylinders are vacuum with perfect electric conducting (PEC) outer surfaces. These cylinders form cylindrical waveguides on either side of the EMI shield. The only difference between the models in Figs. 3 and 4 is the number and size of the pump-out holes. Figure 3 is the original design with $3 \mathrm{~mm}$ diameter holes. Figure 4 has smaller holes, $2 \mathrm{~mm}$ diameter, but has twice as many so that the total cross-sectional area of the holes is $94 \%$ of the total cross-sectional area of the holes in the original design. The approximate matching of the total cross-sectional areas of the holes in the two designs keeps the mechanical pump-out properties of the two EMI shields similar.

A wave port excitation is defined on the end cap of the larger cylinders in Figs. 3 and 4. The wave port excitation effectively models a semi-infinite cylindrical waveguide extending from the wave port surface by using the natural modes of the waveguide for excitation and as boundary conditions. I will denote the wave port on the larger cylinders as "port 1" and the wave port on the smaller cylinders as "port 2". Port 1 is excited by the $T E_{10}$ mode at frequencies from 1 to $5 \mathrm{GHz}$, in $1 \mathrm{GHz}$ increments, with mesh adaptation performed at $5 \mathrm{GHz}$. The wave port boundary condition on port 1 includes the first 20 modes, while the waveport boundary condition on port 2 includes the first 25 modes. The magnitude of the electric field along the centerline is computed for all of the excitation frequencies. One additional simulation is performed, exactly as stated above with Fig. 3, except that the material of the EMI shield is set to vacuum. The additional simulation with the EMI shield set to vacuum includes the effects of only the waveguides and the discontinuity between them, and is used to isolate the shielding effectiveness (SE) of the pump-out holes alone. Figure 5 displays the magnitude of the electric field along the centerline for the models in Figs. 3 and 4 and for the "waveguide only" case.

$\mathrm{SE}$ is computed as follows ${ }^{3}$ :

$$
S E=-20 \log _{10}\left(\frac{|E|}{|E|_{\text {waveguide }}}\right)
$$

where $|E|_{\text {waveguide }}$ is obtained from the simulation with the EMI shield set to vacuum. The resulting values of $\mathrm{SE}$ are displayed for all observation points after the EMI shield in Fig. 6. Figure 7 displays the information from Fig. 6 in a different form. The range from minimum to maximum SE for each frequency is displayed as a broad band. The average SE, taken before the base-ten logarithm, is displayed as a solid line with circular markers.

\footnotetext{
${ }^{3}$ The definition of SE that I use in this report is "for a given external source, the shielding effectiveness is the ratio of electric or magnetic field strength at a point, before and after the placement of the shield in question," from Gabriel Vasilescu, "Chapter 9 - Interference Reduction Methods", Electronic Noise and Interfering Signals: Principles and Applications, Springer, 2005.
} 


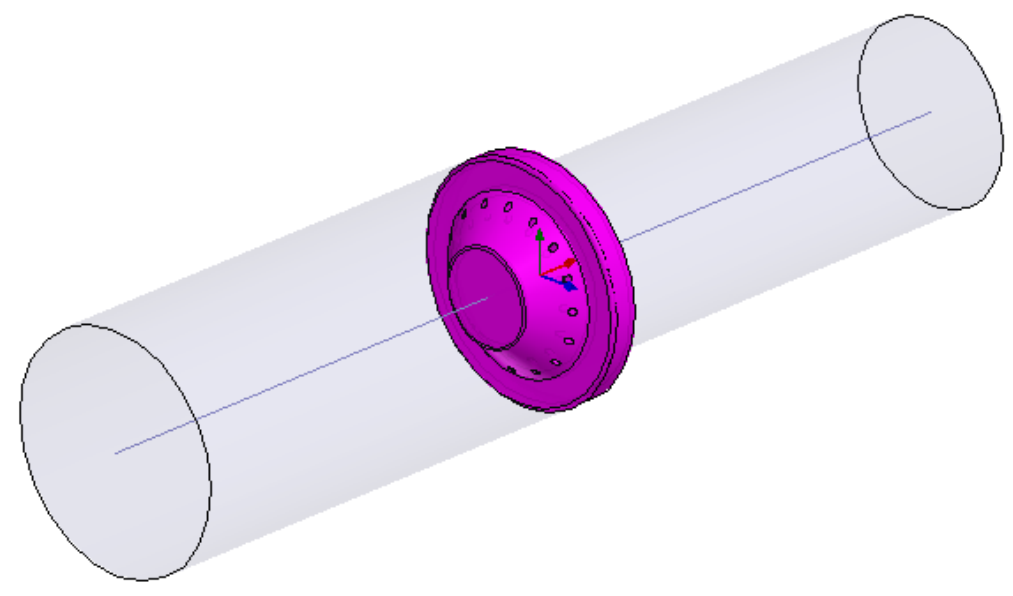

Figure 3: Simulation model for longitudinal incidence on original EMI shield with $3 \mathrm{~mm}$ diameter pump-out holes.

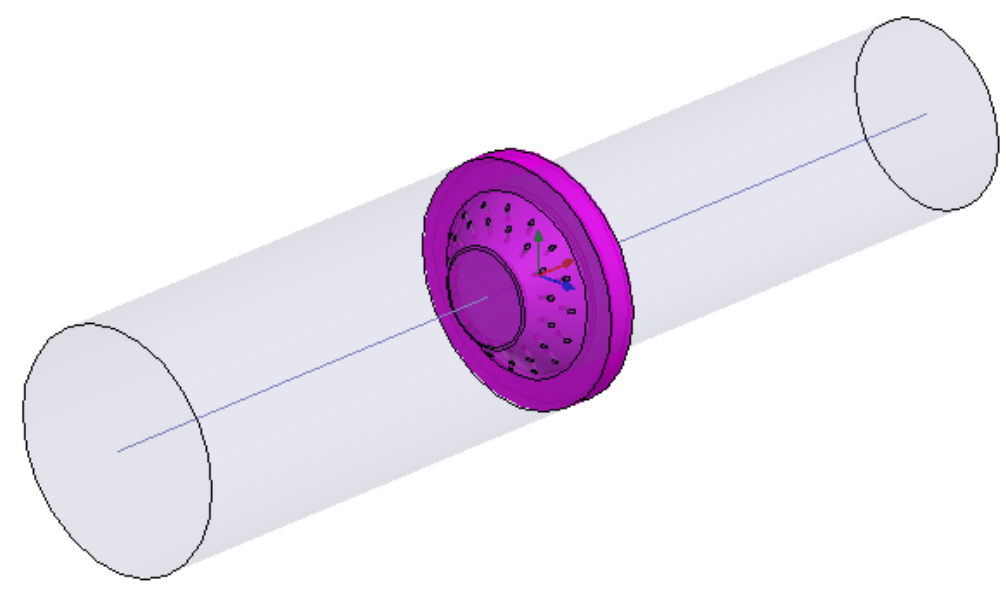

Figure 4: Simulation model for longitudinal incidence on EMI shield with twice as many pump-out holes as in the original. Each pump-out hole is $2 \mathrm{~mm}$ in diameter. 

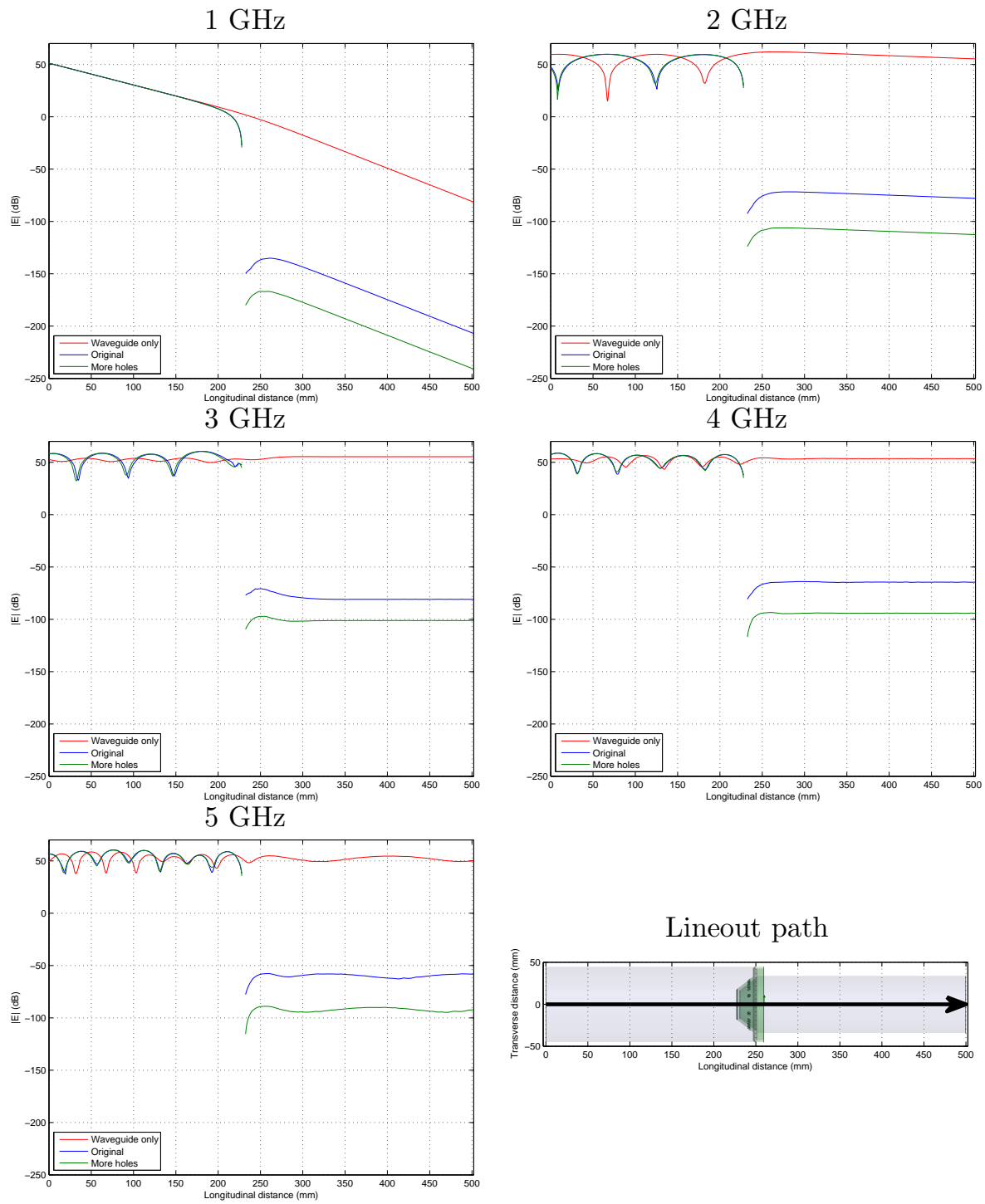

Figure 5: Magnitude of the electric field along the centerline for the models in Figs. 3 and 4. The "Waveguide only" lines include the effects of only the waveguides and the discontinuity between them. 

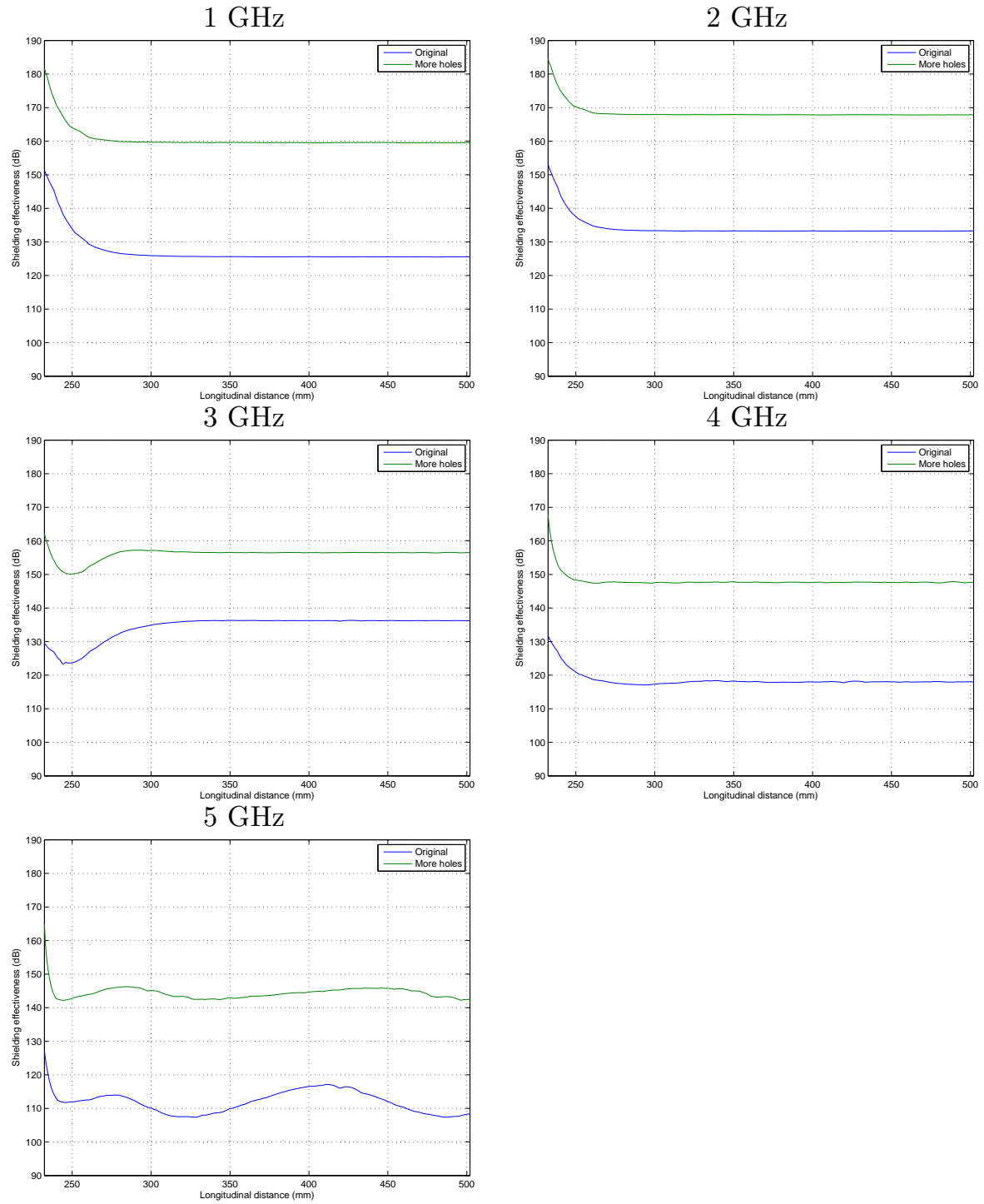

Figure 6: Shielding effectiveness along the centerline for the models in Figs. 3 and 4. The lineout is defined in Fig. 5 


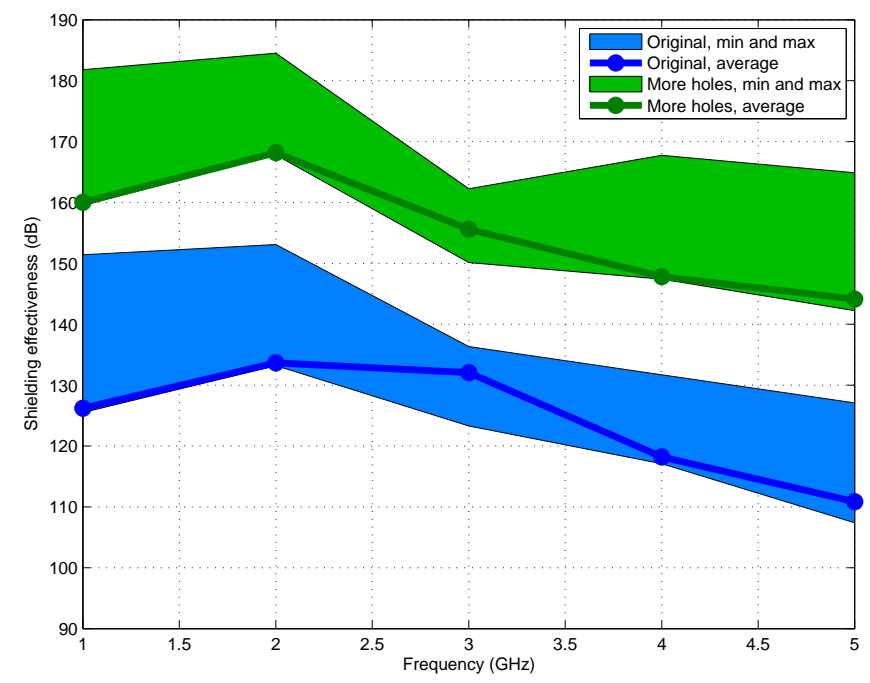

Figure 7: Shielding effectiveness as a function of frequency for longitudinal incidence. The range from minimum to maximum shielding effectiveness for each frequency is displayed as a broad band. The average shielding effectiveness is displayed as a solid line with circular markers.

\section{$2.2 \quad$ Transverse Incidence}

Next, transverse incidence is examined for both the original and alternate designs: Figures 8 and 9 , respectively ${ }^{4}$. The gray cylinders are vacuum with PEC outer surfaces. The squat cylinder, a radial waveguide, has a voltage source defined on the outside face between its circular plates. The voltage source launches an electromagnetic wave into the radial waveguide, which couples through the EMI shield into the smaller cylinder. The smaller cylinder has an absorbing boundary condition on its end cap so that incident waves are partially absorbed. The voltage sources excite the models at frequencies from 1 to $5 \mathrm{GHz}$, in $1 \mathrm{GHz}$ increments. Mesh adaptation is performed at $5 \mathrm{GHz}$. As in Subsection 2.1, one additional simulation is performed, exactly as stated above with Fig. 8, except that the EMI shield is set to vacuum. Figure 10 displays the magnitude of the electric field along the centerline for the models in Figs. 8 and 9 and for the "waveguide only" case.

$\mathrm{SE}$ is calculated using Eqn. 1. Figure 11 displays the resulting $S E$ for the models in Figs. 8 and 9. Figure 12 displays the information from Fig. 11 versus frequency. The range from minimum to maximum SE for each frequency is displayed as a broad band. The average SE, taken before the base-ten logarithm, is displayed as a solid line with circular markers.

\footnotetext{
${ }^{4}$ The EMI shields in the simulation models in Figs. 8 and 9 are identical to the ones in Figs. 3 and 4.
} 


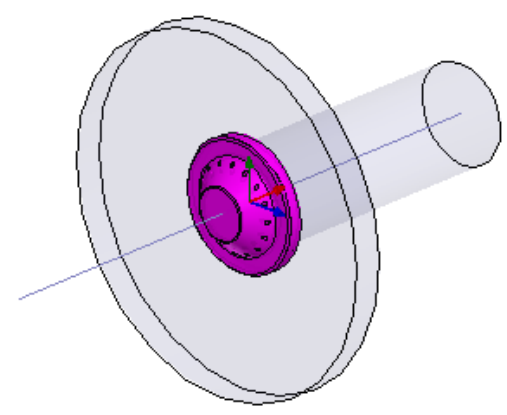

Figure 8: Simulation model for transverse incidence on original EMI shield with $3 \mathrm{~mm}$ diameter pump-out holes.



Figure 9: Simulation model for transverse incidence on EMI shield with twice as many pump-out holes as in the original. Each pump-out hole is $2 \mathrm{~mm}$ in diameter. 

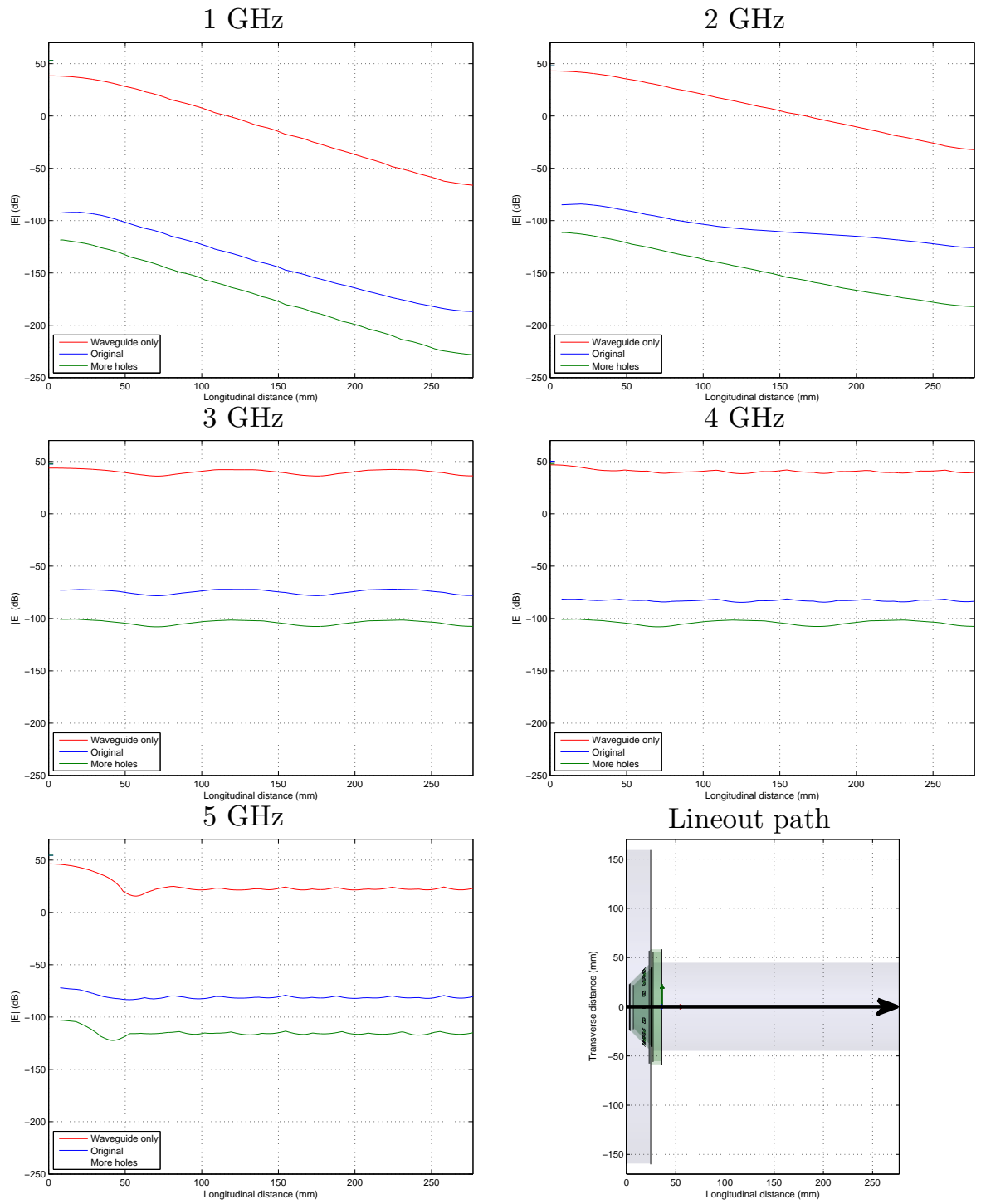

Figure 10: Magnitude of the electric field along the centerline for the models in Figs. 8 and 9. The "Waveguide only" lines include the effects of only the waveguides and the discontinuity between them. 

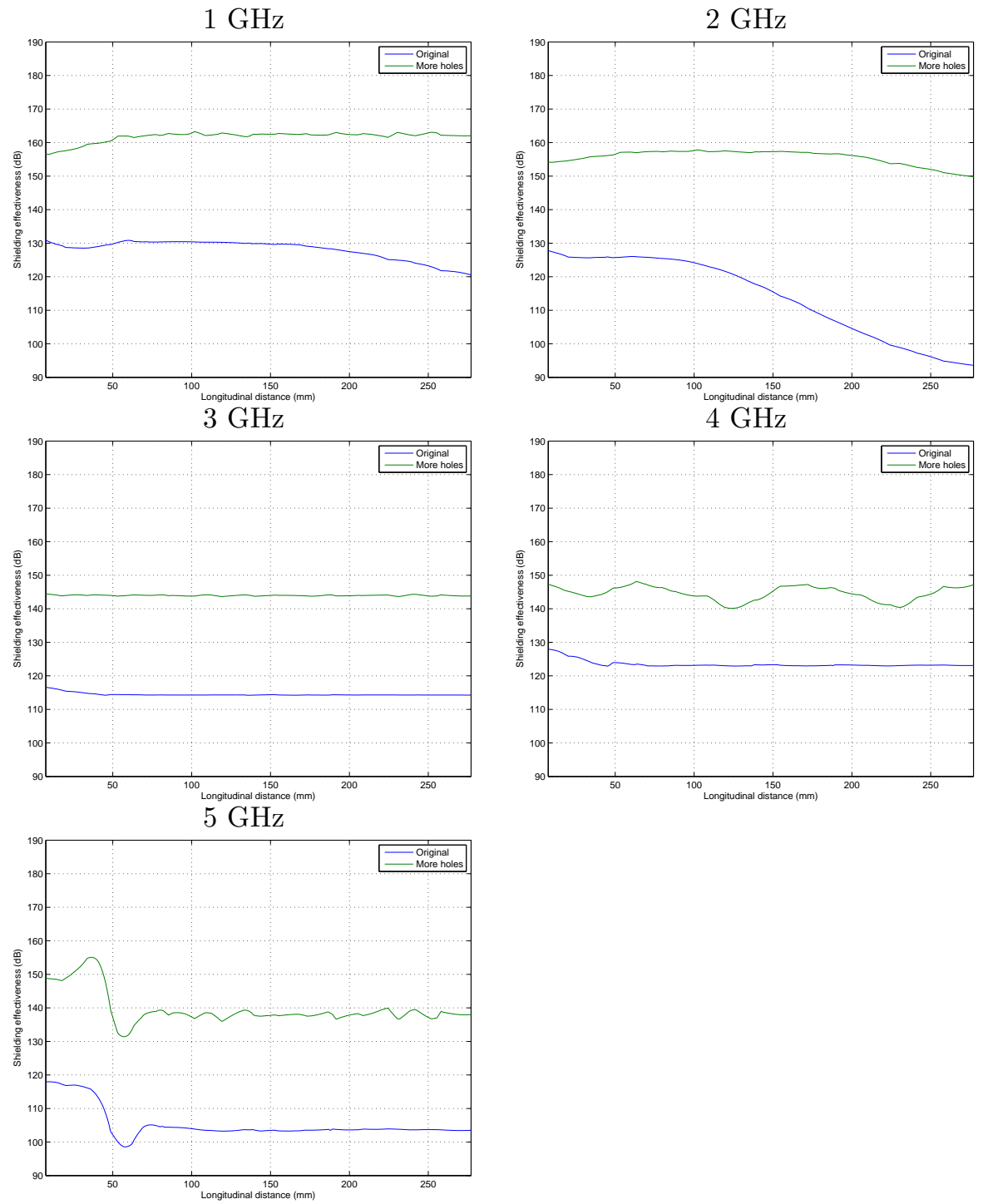

Figure 11: Shielding effectiveness along the centerline for the models in Figs. 8 and 9. The lineout is defined in Fig. 10 


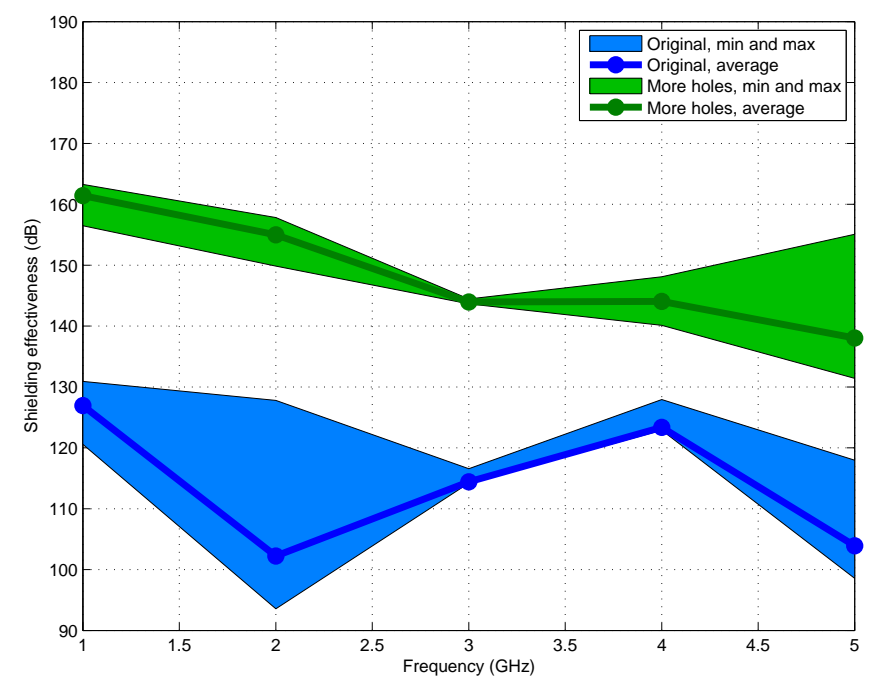

Figure 12: Shielding effectiveness as a function of frequency for transverse incidence. The range from minimum to maximum shielding effectiveness for each frequency is displayed as a broad band. The average shielding effectiveness is displayed as a solid line with circular markers. 